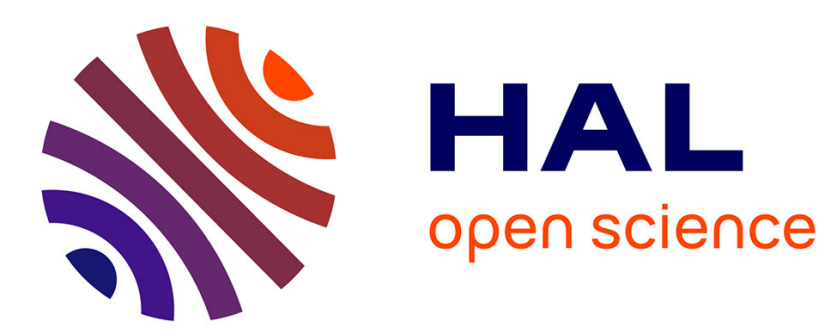

\title{
Dispositif pneumatique d'irradiation auprès du synchrocyclotron de lyon
}

\author{
J. Martin, J. Tousset
}

\section{To cite this version:}

J. Martin, J. Tousset. Dispositif pneumatique d'irradiation auprès du synchrocyclotron de lyon. Revue de Physique Appliquée, 1968, 3 (1), pp.63-66. 10.1051/rphysap:019680030106301 . jpa-00242824

HAL Id: jpa-00242824

https://hal.science/jpa-00242824

Submitted on 1 Jan 1968

HAL is a multi-disciplinary open access archive for the deposit and dissemination of scientific research documents, whether they are published or not. The documents may come from teaching and research institutions in France or abroad, or from public or private research centers.
L'archive ouverte pluridisciplinaire HAL, est destinée au dépôt et à la diffusion de documents scientifiques de niveau recherche, publiés ou non, émanant des établissements d'enseignement et de recherche français ou étrangers, des laboratoires publics ou privés. 
La puissance électrique du convertisseur reste remarquablement stable pendant les premiers essais qui ont duré environ 20 heures.

Conclusion. - Le générateur thermoélectrique tel qu'il a été conçu et réalisé vise principalement à montrer la possibilité d'utiliser certains matériaux semiconducteurs au-delà de leur point de fusion en vue de la conversion de la chaleur. Les premiers résultats obtenus apparaissent prometteurs, bien que certains problèmes technologiques tels que la résistance de contact, l'isolement thermique restent encore à résoudre en vue d'améliorer les performances du convertisseur.

\title{
BIBLIOGRAPHIE
}

[1] Marcus (R. J.) et KelLy (C. M.), Thermoelectric Materials and Devices, édité par Cadoff et Miller, p. 185, Reinhold Publishing Corporation, 1960.

[2] Cútier (M.) et Malion (C. E.), J. Appl. Physics, 1965, 36, 201.
[3] Nguyen Van Dong et Pham Ngu Tung, C. R. Acad. Sc. Paris, 1966, 262, 63; C. R. Acad. Sc. Paris, 1966, 262, 1347.

\section{DISPOSITIF PNEUMATIQUE D'IRRADIATION AUPRÈS DU SYNGHROGYGLOTRON DE LYON}

\author{
Par J. MARTIN et J. TOUSSET, \\ Institut de Physique Nucléaire de Lyon. \\ (Reçu le 23 novembre 196\%.)
}

\begin{abstract}
Résumé. - Un dispositif pneumatique d'irradiation auprès d'un synchrocyclotron est décrit, comportant la possibilité d'irradiation dans le vide d'échantillons mis en place sur cartouche porteuse dans un laboratoire de chimie situé à 140 mètres de l'accélérateur et leur retour en moins d'une minute après la fin de l'irradiation.
\end{abstract}

Abstract. - A pneumatic tube device for rapid, automatic positioning and removal of targets in the vacuum chamber of the external beam of a synchrocyclotron is described. The target can be returned to the radiochemical laboratory, a distance of 140 meters, within one minute after the end of the irradiation.

I. Introduction. - Les études de chimie nucléaire qui se développent auprès d'accélérateurs, que ce soient le tracé de fonctions d'excitation, la production de radio-isotopes à courte période, l'analyse par activation, nécessitent souvent de brèves et fréquentes irradiations. Outre la difficulté et l'exposition aux radiations que représentent les retraits manuels d'échantillons irradiés, la perte de temps qui en résulte, tant pour la durée d'occupation de la machine que pour l'activité des produits à courte période, rend fort utile l'élaboration d'un dispositif semi-automatique d'irradiation qui permet la marche quasi continue de l'accélérateur.

Les caractéristiques d'un tel appareil doivent être la sécurité, la rapidité et la possibilité d'irradiation des échantillons dans le vide. Certains dispositifs ont déjà été rapportés [1] ou sont en cours d'étude [2].
Nous décrivons ci-dessous l'ensemble réalisé à l'Institut de Physique Nucléaire de Lyon, dont le fonctionnement depuis un an est très satisfaisant.

On peut naturellement distinguer dans le système deux parties : les cartouches porteuses et l'installation fixe.

II. Les cartouches d'irradiation. - Elles sont de deux modèles, ce qui justifie le grand diamètre du tube : $100 \mathrm{~mm}$. Dans un cas, en effet, quatre échantillons (de $20 \mathrm{~mm}$ de diamètre maximal) sont irradiés, fixés sur un porte-échantillon tournant qui les présente tour à tour au faisceau, ce qui suppose un axe de rotation excentré, donc un décalage par rapport au faisceau pour un tube porteur de petite section. Cette solution interdirait l'utilisation aisée du second type très simple de cartouches, à échantillon unique 
et fixe (ou empilement) dont le positionnement en irradiation aurait été difficile. La solution du tube cylindrique assez gros, centré sur l'axe du faisceau, qui peut englober le système rotatif excentré et conserver la symétrie axiale dans le cas de l'échantillon unique, semble la plus judicieuse.

Les cartouches du second type sont réalisées en duralumin. Une représentation en est faite sur la figure 1. La face avant comporte un joint « quad-ring »

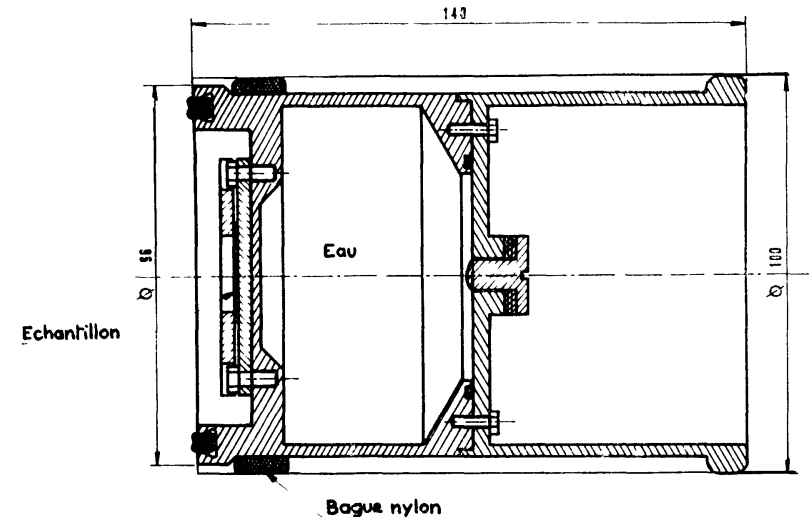

FIG. 1. - Cartouche à échantillon fixe.

qui assure une excellente étanchéité en position d'irradiation. L'échantillon, fixé dans l'axe de la cartouche par une rondelle formant diaphragme, est refroidi par contact avec un petit réservoir de $100 \mathrm{~cm}^{3}$ d'eau, volant thermique suffisant pour nos expériences au cours desquelles l'intensité du faisceau ne dépasse pas $1 \mu \mathrm{A}$ sous $54 \mathrm{MeV}$ en $\alpha$ et $27 \mathrm{MeV}$ en deutons. Une bague de nylon montée à l'avant sur le pourtour permet un glissement correct. La masse d'une cartouche de ce type est de $920 \mathrm{~g}$ environ.

Les cartouches à échantillons tournants sont réalisées en magnésium. Elles comportent, comme les précédentes, un joint «quad-ring » et une bague de glissement en nylon. Une coupe est faite sur la figure 2. Le porte-échantillon tournant rempli d'eau

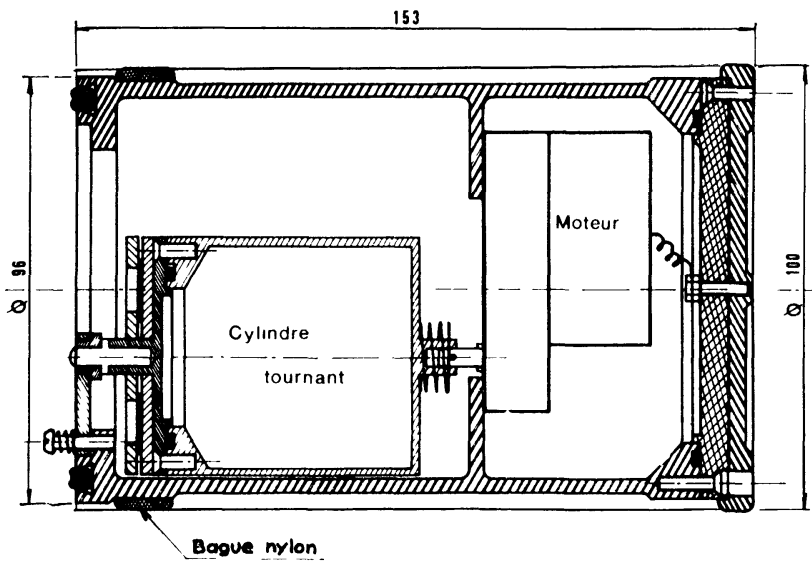

FIG. 2. - Cartouche à échantillons tournants. et monté sur amortisseurs est entraîné à la vitesse de $60 \mathrm{tr} / \mathrm{mn}$ par un petit moteur incorporé dont l'alimentation est assurée par contact circulaire sur la face arrière. La masse de cet ensemble est de l'ordre de $1000 \mathrm{~g}$.

III. L'installation fixe. - Elle comprend, outre le tube, la tête d'irradiation raccordée à la voie dite « $\mathrm{O}$ » du synchrocyclotron (voir fig. 3) et un sas de réception ventilé.

Pour des raisons d'exploitation en commun d'appareils de mesures (sélecteur, détecteur...) utilisés avec

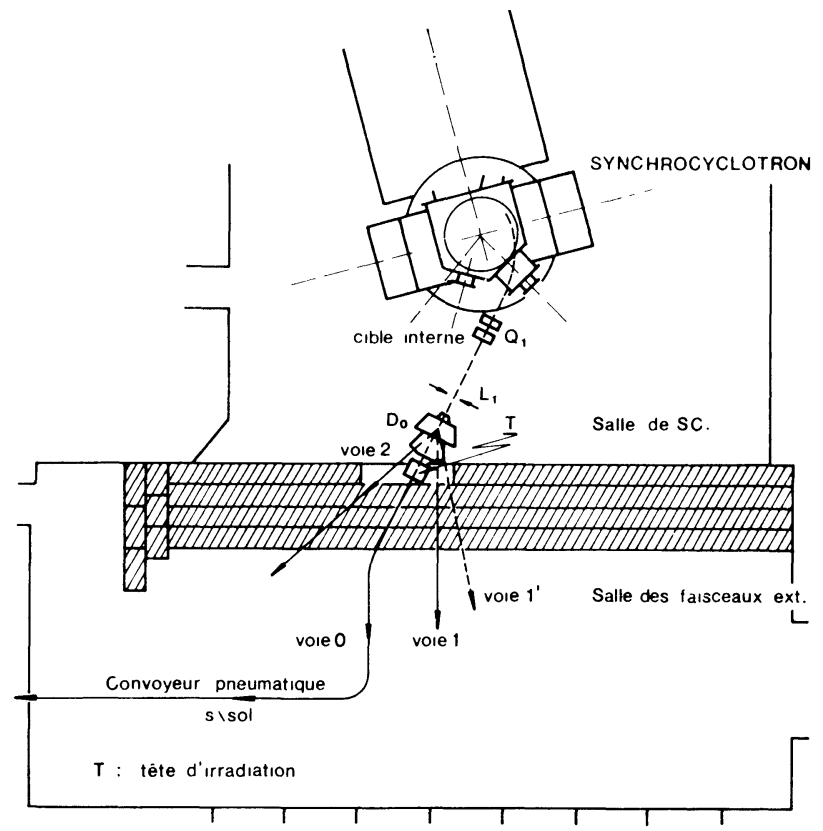

FIG. 3. - Implantation du convoyeur sur les voies de sortie.

un autre convoyeur [3], le trajet est assez long (140 m) et doit emprunter des galeries techniques à multiples courbes. Les facteurs prix, poids, raccords et rayons de courbure ont imposé le tube de chlorure de polyvinyle dur $(102,8-110 \mathrm{~mm}, 1,685 \mathrm{~kg} / \mathrm{m})$. Les rayons de courbure sont de 1,50 mètre et les raccords sont réalisés à l'aide de manchons collés. Certains éléments sont démontables. L'étanchéité est alors assurée par joint torique. La tête d'irradiation est partiellement représentée sur la figure 4 avec une cartouche en place. Cette dernière est alors horizontale, dans l'axe du faisceau. Centrée par l'intermédiaire d'un cône, elle agit sur des microcontacts de contrôle. Son joint vient plaquer sur une surface plane en acier inoxydable, qui fait partie d'une pièce cylindrique à deux piquages, dont l'un sert au pompage primaire, l'autre à la propulsion pneumatique. Le léger choc à l'arrivée de la cartouche justifie un montage de l'ensemble sur amortisseur.

Une vanne à tiroir électropneumatique assure la 
liaison de cette tête d'irradiation avec la voie de sortie du synchrocyclotron. Entre cette vanne et la cartouche, dans l'axe du faisceau, se trouvent les organes suivants :

- Une chambre à sorties multiples permettant divers contrôles,

- Une boîte à écran de tantale,

- Une seconde vanne à tiroir électropneumatique,

- Un diaphragme,

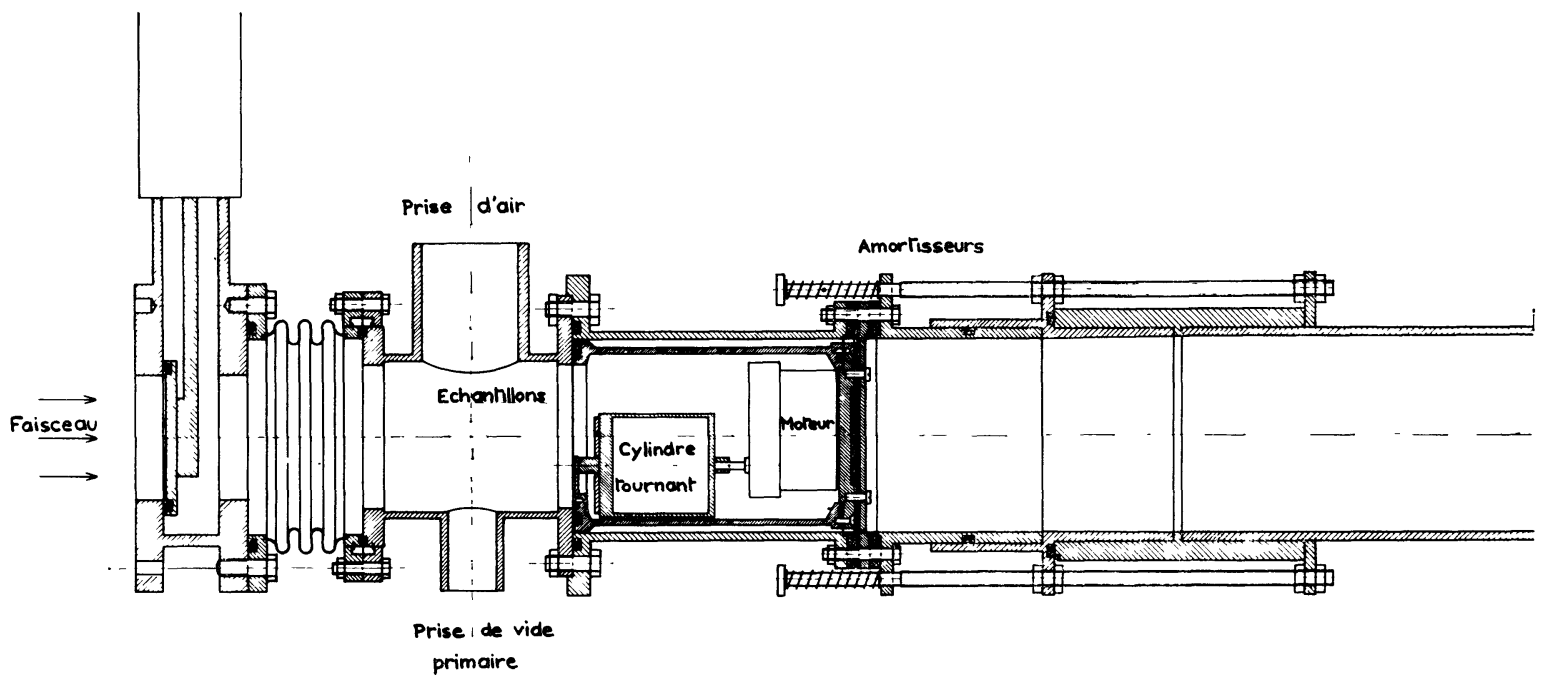

FIG. 4. - Tête d'irradiation avec cartouche en place.

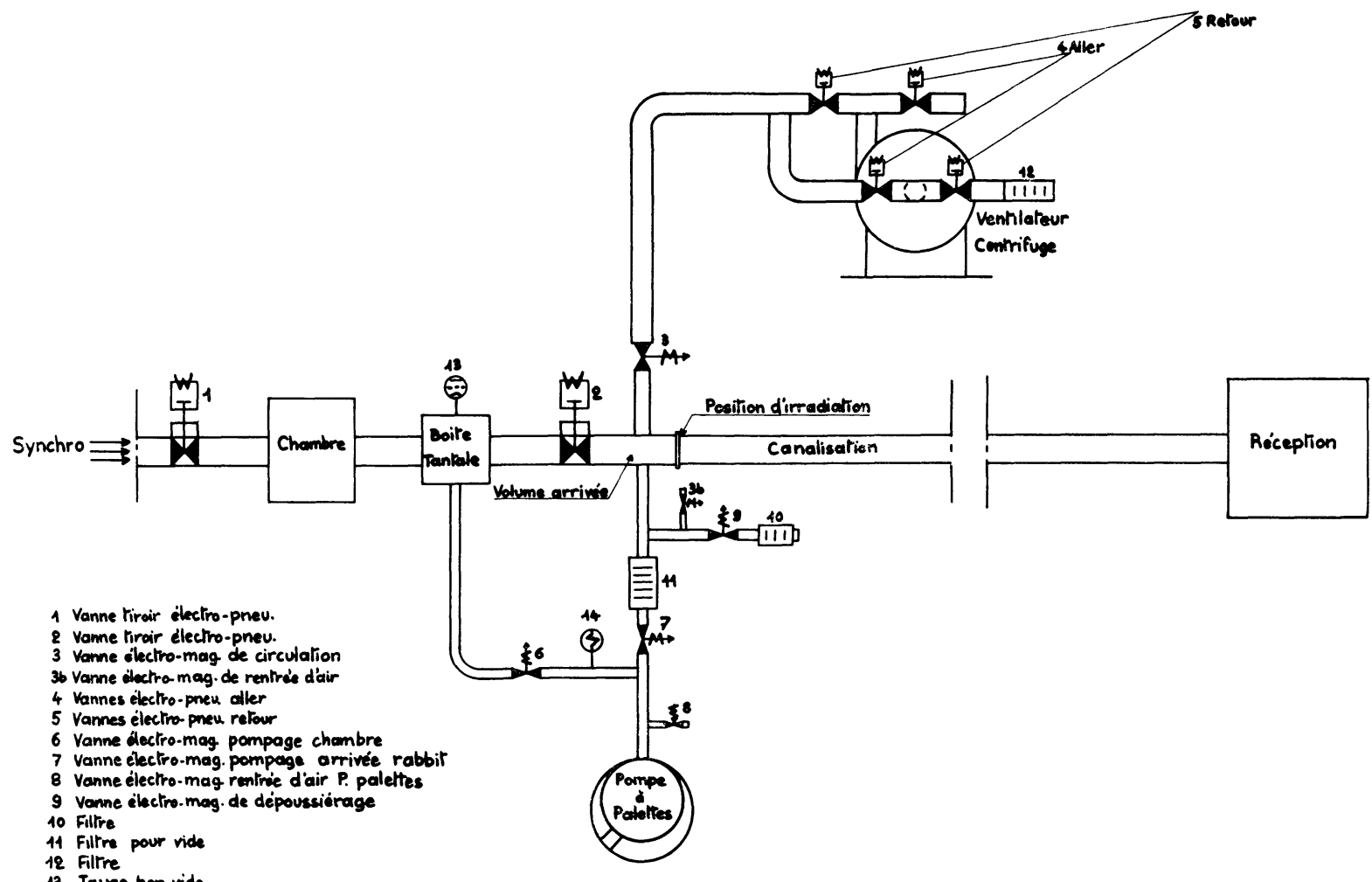

FIG. 5. - Schéma de principe.
- Un tombac qui sert à compenser la très légère élongation produite par le fonctionnement des amortisseurs.

Le support réglable de cet ensemble abrite le groupe de pompage comprenant pompes, vannes électromagnétiquєs, filtres et têtes de jauge.

A l'autre extrémité, la cartouche arrive à son retour dans un berceau-frein situé dans une boîte à gants ventilée. 
IV. La propulsion (fig. 5). - La propulsion est assurée par aspiration à l'aller et par pression au retour grâce à un ventilateur centrifuge (pression $400 \mathrm{~mm} \mathrm{CE}$, débit $0,04 \mathrm{~m}^{3} / \mathrm{s}$ ). Un jeu de quatre vannes de gros diamètre, à commande par vérins pneumatiques, réalise la permutation nécessaire. Une autre vanne à commande électromagnétique, située à l'entrée de la canalisation, conditionne l'avance de la cartouche. Toutes les manœuvres sont dirigées par un combinateur dont le programme est affiché une fois pour toutes.

V. Fonctionnement. - On engage la cartouche dans le tube au niveau du sas de réception et le combinateur est enclenché. L'aspiration se trouve coupée automatiquement avant l'arrivée; à titre de sécurité, un détecteur de passage double cette fonction et un système de retard réglable associé à cet ensemble permet d'obtenir une arrivée précise. La cartouche arrivant en position d'irradiation, un voyant lumineux s'éclaire et une brève opération de dépoussiérage se déclenche, dont le but est d'éliminer d'éventuelles poussières déposées sur la cartouche pendant son trajet. Ceci est réalisé par une circulation d'air pulsé dans le petit volume situé devant la cartouche. Un vide primaire s'établit ensuite et son obtention autorise l'ouverture de la vanne tiroir la plus proche qui met en communication avec le vide secondaire de la chambre d'accélération.
L'irradiation débute avec l'escamotage de la plaque de tantale, lequel provoque la rotation des échantillons dans le cas du type correspondant de cartouche. Lorsque le temps affiché pour l'irradiation est écoulé, un signal électrique assure les multiples fonctions suivantes :

- Fermeture de la plaque de tantale,

- Fermeture de la vanne d'isolement,

- Mise en route du combinateur commandant le retour du convoyeur,

- Mise en route du chronomètre de transit.

La cartouche est ralentie, comme à l'aller, pour arriver en douceur dans le sas de réception. Plusieurs sécurités de vide interdisent toute rentrée d'air accidentelle sur l'accélérateur et toute autre fausse manœuvre. Des compteurs numériques notent les nombres et durées d'irradiations.

VI. Performances. - Le temps nécessaire pour effectuer le trajet de 140 mètres est, compte tenu des ralentissements en bout de course, de l'ordre de 40 secondes. Le dépoussiérage est fixé à une minute, l'établissement du vide dure de 3 à 4 minutes. L'irradiation peut donc commencer 5 minutes après l'envoi et l'échantillon est à la disposition de l'opérateur moins d'une minute après l'irradiation.

Nous tenons à remercier $\mathrm{H}$. Bonin pour la compétence et l'efficacité de sa collaboration.

\section{BIBLIOGRAPHIE}

[1] Aspects pratiques de l'analyse par activation au moyen de particules chargées, Rapport Euratom EUR-2957, d.f.e. (juin 1965).

[2] Aspects pratiques de l'analyse par activation au moyen de particules chargées. Colloque Euratom, Liège, septembre 1967.

[3] Martin (J.) et Tousset (J.), Rev. Physique Appl., $1966,1,243$. 